JIRSS (2018)

Vol. 17, No. 1, pp 33-47

DOI: $10.29252 /$ jirss.17.1.33

\title{
Bayes, E-Bayes and Robust Bayes Premium Estimation and Pre- diction under the Squared Log Error Loss Function
}

\author{
Azadeh Kiapour \\ Department of Statistics, Babol Branch, Islamic Azad University, Babol, Iran \\ Received: 23/11/2016, Revision received: 22/08/2017, Published online: 19/05/2018
}

\begin{abstract}
In risk analysis based on Bayesian framework, premium calculation requires specification of a prior distribution for the risk parameter in the heterogeneous portfolio. When the prior knowledge is vague, the E-Bayesian and robust Bayesian analysis can be used to handle the uncertainty in specifying the prior distribution by considering a class of priors instead of a single prior. In this paper, we study the E-Bayes and robust Bayes premium estimation and prediction in exponential model under the squared log error loss function. A prequential analysis in a simulation study is carried out to compare the proposed predictors. Finally, a real data example is included for illustrating the results.
\end{abstract}

Keywords. Bayes premium, Class of priors, E-Bayes premium, Exponential distribution, Robust Bayes premium.

MSC: 62F15; 62P05.

\section{Introduction}

In actuarial studies, the future premium of a policyholder is computed on the basis of past claims size using some premium principles. A premium calculation principle is a function that assigns a normally loaded premium to the underlying distribution of

$\overline{\text { Azadeh Kiapour (kiapour@baboliau.ac.ir). }}$ 
claim size. In practice, the premium can be calculated when the distribution of claim size is known. Suppose that the distribution of the claim size is specified up to an unknown parameter $\theta$, thus the risk premium will be denoted by $P(\theta)$.

In order to obtain an appropriate estimate of $P(\theta)$ or to predict the future value of the claim size, various methodologies have been proposed in the literature. One of the most interesting methodology is based on the Bayesian theory. The standard Bayesian analysis has been used in the risk theory and actuarial studies by several people, e.g., Heilmann (1989), Goovaerts et al. (1990) and Klugman (1992), among others. In the Bayesian method, the practitioner usually chooses a prior distribution over the parameter space. However, in most cases, the practitioner is unwilling or unable to choose a specific prior distribution. However, he/she may be able to restrict the prior to a class of prior distributions that are suitable for quantifying the actuary's uncertainty. E-Bayesian and robust Bayesian analyses deal with such problems and study the effect of changing a prior within the class of prior distributions, for some quantity such as the posterior or Bayes risk. Therefore, it is of interest to study the behavior of the premium under the class of priors, by using E-Bayesian and some robust Bayesian criteria.

The goal of the E-Bayesian method is estimating an unknown parameter or predicting a future value of a sequence of random variables by specifying a prior distribution on prior hayperparameter(s). The E-Bayesian analysis is investigated by Han (1997, 2007, 2009, 2011) and Jaheen and Okasha (2011). We shall extend E-Bayes estimation (prediction) to E-Bayes premium estimation (prediction) in actuarial framwork.

In robust Bayesian analysis, there are several methods to obtain optimal rules for estimating an unknown parameter or predicting a future value of a sequence of random variables: The $\Gamma$-minimax rules (e.g., Berger, 1984), the conditional $\Gamma$-minimax rules (e.g., Betro and Ruggeri, 1992), the posterior regret $\Gamma$-minimax rules (e.g., Rios Insua et al., 1995), the least sensitive rules (Arias-Nicolas et al., 2009) and the most stable rules (Meczarski, 1993), which are connected with the problem of finding estimator or predictor with the smallest supremum of Bayes risk, posterior risk, posterior regret, sensitivity and the smallest oscillation of the posterior risk, respectively, when the prior runs over the class $\Gamma$. The robust Bayesian estimation (prediction) has broad applications in actuarial sciences. For example, Rios et al. (1999), Gomez-Deniez et al. (2006) and Boratynska (2008), among others, used the robust Bayesian methodology to estimate premiums or predict the future value of the claim size under different loss functions and different risk models.

In this paper, we use the squared log error loss (SLEL) function for estimating $P(\theta)$ and predicting the future value of the claim size. We study the E-Bayesian premium and also the robust Bayesian premium estimation (prediction) including the posterior 
regret $\Gamma$-minimax (PRGM), the conditional $\Gamma$-minimax (CGM), the least sensitive (LS) and the most stable (MS) methods. To do this, we review some usual risk premiums and derive the Bayes premium in Section 2. We provide E-Bayes and robust Bayes premium estimators under a class of conjugate prior distributions in Section 3. Bayes, E-Bayes and robust Bayes premium predictors of the future claim sizes are obtained in Section 4. In Section 5, a prequential analysis using a simulation is conducted to compare the proposed Bayes, E-Bayes and robust Bayes premium predictors. The proposed methods are applied to a real data-set in Section 6. Finally, we end the paper with some concluding remarks.

\section{Risk premium}

Suppose $X$ is a random variable with probability density function (p.d.f.) $f(x \mid \theta)$ which denotes the claim size or loss amount of one contract in one period, where $\theta \in \Theta$ is the unknown parameter of the model. Let $\pi(\theta)$ denote the prior distribution of $\theta$. A premium calculation principle (Heilmann, 1989) is a function that assigns a real number to risk $X$. Let $L: R^{2} \longrightarrow R$ be a loss function that assigns to any $(x, P) \in R^{2}$ the loss sustained by a decision maker who takes the action $P$ and is faced with the outcome $x$ of a random experience. The unknown risk premium $P(\theta)$ can be obtained by minimizing the expected loss $E[L(X, P)]$ with respect to (w.r.t.) $\theta$. Let $X$ be the claim size and, given $\theta$, suppose it has an $\operatorname{EXP}(\theta)$-distribution with p.d.f.

$$
f(x \mid \theta)=\theta e^{-\theta x}, \quad x>0, \quad \theta>0 .
$$

The risk premium $P(\theta)$ under some loss functions are given in the sequel:

(i) When $L_{1}(x, P)=(\ln x-\ln p)^{2}, x>0, p>0$ and $P(X>0)=1$,

$$
P_{1}(\theta)=e^{E(\ln X \mid \theta)}=\frac{e^{\psi(1)}}{\theta}=\frac{u_{1}}{\theta}
$$

where $\psi(v)=\frac{d}{d(v)} \ln \Gamma(v)=\frac{\Gamma^{\prime}(v)}{\Gamma(v)}$ is the digamma function, $u_{1}=e^{\psi(1)}$ and $\psi(1)=$ -0.5772 , which can be computed using command digamma(1) in $\mathrm{R}$ version 3.1.2.

(ii) When $L_{2}(x, P)=(x-p)^{2}$,

$$
P_{2}(\theta)=E(X \mid \theta)=\frac{1}{\theta}=\frac{u_{2}}{\theta}
$$

which is known as the net premium principle, where $u_{2}=1$. 
(iii) When $L_{3}(x, P)=x(x-p)^{2}$,

$$
P_{3}(\theta)=\frac{E\left(X^{2} \mid \theta\right)}{E(X \mid \theta)}=\frac{2}{\theta}=\frac{u_{3}}{\theta}
$$

which is known as the variance premium principle, where $u_{3}=2$.

(iv) When $L_{4}(x, P)=\frac{x}{P}-\ln \frac{x}{P}-1, x>0, p>0$ and $P(X>0)=1$,

$$
P_{4}(\theta)=E(X \mid \theta)=\frac{1}{\theta}=\frac{u_{4}}{\theta},
$$

where $u_{4}=1$.

Note that the risk premium $P(\theta)$ under the proposed loss functions is of the form $u / \theta$. The estimation of the risk premium $P(\theta)$ under the square error loss (SEL) or linearexponential (LINEX) loss has been studied in the literature, see Heilmann (1989). Most of the previously studied loss functions in the risk theory are either symmetric or are not suitable for the estimation of the scale parameter $\theta$. However, in practice, the penalty for underestimation or overestimation of $\theta$ does not necessarily have to be the same. Furthermore, when the risk premium $P(\theta)$ is a linear function of the scale parameter $\theta$, more suitable loss functions are those which are scale invariant. This motivates us to consider a scale-invariant loss function. Brown (1968) proposed the SLEL function of the form

$$
L(P(\theta), P)=(\ln P-\ln P(\theta))^{2}=\left[\ln \frac{P}{P(\theta)}\right]^{2},
$$

where $P$ is an estimate of $P(\theta)$ based on the observed claim $x$ and both $P(\theta)$ and $P$

are positive. The loss function (2.2) is asymmetric and convex when $\Delta=\frac{P(\theta)}{P} \leq e$ and concave otherwise, but has a unique minimum at $\Delta=1$. This loss penalizes heavily underestimation, so, it is suitable for the estimation of the risk premium $P(\theta)$. The SLEL function is considered by researchers in some estimation and prediction problems, see Sanjari Farsipour and Zakerzadeh (2005), Kiapour and Nematollahi (2011) and Naghizadeh Qomi and Barmodeh (2015).

\section{Estimation Strategies of Risk Premium}

\subsection{The Bayes Premium}

Let $X_{i}$ be the claim size in year $i=1,2, \ldots$. Suppose $X_{1}, X_{2}, \ldots$ be a sequence of random claim sizes and $\mathbf{X}^{n}=\left(X_{1}, \ldots, X_{n}\right)$. Assume that given $\theta, X_{1}, X_{2}, \ldots$ are stochastically 
independent and identically distributed. To calculate the Bayes premium, we use both the prior information about the parameter $\theta$ and the actual loss experience observed during the policy period, $\mathbf{x}^{n}=\left(x_{1}, \ldots, x_{n}\right)$ from $\mathbf{X}^{n}$. Our objective is to estimate $P(\theta)$ under the loss function (2.2) by $P \in D$, where $D$ is the class of all decisions having finite risks. The Bayes premium of $P(\theta)$ is obtained by minimizing the posterior risk

$$
\begin{aligned}
\rho(\pi, P) & =E\left[L(P(\theta), P) \mid \mathbf{X}^{n}=\mathbf{x}^{n}\right] \\
& =\frac{\int_{\Theta} L(P(\theta), P) f_{\theta}\left(\mathbf{x}^{n}\right) \pi(\theta) d v(\theta)}{\int_{\Theta} f_{\theta}\left(\mathbf{x}^{n}\right) \pi(\theta) d v(\theta)} \\
& =\ln ^{2} P+E\left[\ln ^{2} P(\theta) \mid \mathbf{x}^{n}\right]-2 \ln P E\left[\ln P(\theta) \mid \mathbf{x}^{n}\right],
\end{aligned}
$$

over $P \in D$, where $\pi(\theta)$ is a prior distribution w.r.t. some $\sigma$-finite measure $v$. A straightforward calculation shows that the Bayes premium under the loss function (2.2) is given by

$$
P^{B}\left(\mathbf{x}^{n}\right)=e^{E\left[\ln P(\theta) \mid \mathbf{x}^{n}\right]}
$$

Suppose that, given $\theta$, the claim size $X_{i}, i=1,2, \ldots, n$, has an $\operatorname{EXP}(\theta)$-distribution. Assume that the prior distribution of $\theta$ is $\operatorname{Gamma}(\alpha, \beta)$ with p.d.f.

$$
\pi_{\alpha, \beta}(\theta)=\frac{\beta^{\alpha}}{\Gamma(\alpha)} \theta^{\alpha-1} e^{-\beta \theta}, \quad \alpha>0, \beta>0, \theta>0 .
$$

If $\mathbf{X}^{n}=\mathbf{x}^{n}$, then the posterior distribution is again $\operatorname{Gamma}(n+\alpha, T+\beta)$ where $T=\sum_{i=1}^{n} X_{i}$. Following Sanjari Farsipour and Zakerzadeh (2005), $E\left[\ln \theta \mid \mathbf{x}^{n}\right]=\psi(n+\alpha)-\ln (T+\beta)$. Now, consider the risk premium of the form $P(\theta)=\frac{u}{\theta}$ where $u$ is a constant. Using (3.2), the Bayes premium under the loss function (2.2) is given by

$$
P^{B}\left(\mathbf{x}^{n}\right)=e^{E\left[\ln P(\theta) \mid \mathbf{x}^{n}\right]}=u(T+\beta) e^{-\psi(n+\alpha)} .
$$

\subsection{E-Bayes Premium Estimator}

The E-Bayesian premium estimation is the expectation of the Bayesian premium estimation for all hyperparameters. Here we introduce and extend the E-Bayesian problem into estimation and prediction premium problem.

According to Han (1997), hyperparameters $\alpha$ and $\beta$ in (3.3) should be chosen so that it is guaranteed that $\pi_{\alpha, \beta}(\theta)$ is a decreasing function of $\theta$. Then, we have $0<\alpha<1$ and $\beta>0$ due to $\frac{d \pi_{\alpha, \beta}(\theta)}{d \theta}<0$. When $\alpha=1, \pi$ is a decreasing function of $\theta$. Following Berger 
(1985), $\beta$ should not be too big while $\alpha=1$. It is better to choose $0<\beta<c$. Then, the prior density of $\theta$ becomes

$$
\pi_{\beta}(\theta)=\beta e^{-\beta \theta} .
$$

The E-Bayes estimator is defined as

$$
P^{E B}\left(\mathbf{x}^{n}\right)=\int_{D_{\beta}} P^{B}\left(\mathbf{x}^{n}\right) \pi(\beta) d \beta,
$$

where $P^{B}\left(\mathbf{x}^{n}\right)$ is the Bayes premium estimate (3.4) with $\alpha=1, \pi(\beta)$ is a prior density and $D_{\beta}$ is the domain of $\beta$.

According to Han (1997), we consider three prior distributions of the hyperparameter $\beta$ for E-Bayesian premium estimation of $P(\theta)$ as

$$
\begin{aligned}
& \pi_{1}(\beta)=\frac{2(c-\beta)}{c^{2}}, \quad \alpha=1,0<\beta<c, \\
& \pi_{2}(\beta)=\frac{1}{c^{\prime}} \quad \alpha=1,0<\beta<c, \\
& \pi_{3}(\beta)=\frac{2 \beta}{c^{2}}, \quad \alpha=1,0<\beta<c .
\end{aligned}
$$

In the following theorem, we obtain the E-Bayes premium estimators of $P(\theta)$ under the loss function (2.2) and prior distributions given in (3.7).

Theorem 3.1. Let $X_{1}, X_{2}, \ldots$ be a sequence of random variables which are conditionally independent given $\theta$, and suppose that $X_{i}$ given $\theta$ has $\operatorname{EXP}(\theta)$-distribution. Suppose that $X^{n}=x^{n}$. Then, the E-Bayes premium estimators of $P(\theta)=\frac{u}{\theta}$ under three prior distributions in (3.7) are equal to

$$
\begin{aligned}
& P^{E B 1}\left(x^{n}\right)=u e^{-\psi(n+1)}\left[\frac{c}{3}+T\right], \\
& P^{E B 2}\left(x^{n}\right)=u e^{-\psi(n+1)}\left[\frac{c}{2}+T\right], \\
& P^{E B 3}\left(x^{n}\right)=u e^{-\psi(n+1)}\left[\frac{2 c}{3}+T\right] .
\end{aligned}
$$

proof. For $\pi_{1}(\beta)$, the E-Bayes premium estimate under the loss function (2.2) is obtained as

$$
P^{E B 1}\left(\mathbf{x}^{n}\right)=\int_{D_{\beta}} P^{B}\left(\mathbf{x}^{n}\right) \pi_{1}(\beta) d \beta=\int_{0}^{c} u(\beta+T) e^{-\psi(n+1)} \frac{2(c-\beta)}{c^{2}} d \beta
$$




$$
=u e^{-\psi(n+1)}\left[\frac{c}{3}+T\right] .
$$

Similarly, if the prior distribution of $\beta$ are given by $\pi_{2}(\beta)$ and $\pi_{3}(\beta)$, then, the corresponding E-Bayes premium estimates under the loss function (2.2) will be, respectively, as

$$
\begin{gathered}
P^{E B 2}\left(\mathbf{x}^{n}\right)=\int_{D_{\beta}} P^{B}\left(\mathbf{x}^{n}\right) \pi_{2}(\beta) d \beta=u e^{-\psi(n+1)}\left[\frac{c}{2}+T\right], \\
P^{E B 3}\left(\mathbf{x}^{n}\right)=\int_{D_{\beta}} P^{B}\left(\mathbf{x}^{n}\right) \pi_{3}(\beta) d \beta=u e^{-\psi(n+1)}\left[\frac{2 c}{3}+T\right] .
\end{gathered}
$$

\subsection{Robust Bayes Premium Estimator}

In this subsection, we study the problem of estimating $P(\theta)$ under some useful criteria for the $\operatorname{EXP}(\theta)$ model under the loss function (2.2). In the sequel, we recall the definitions of several optimality criteria and optimal estimators in the robust Bayesian analysis.

Definition 3.1. (Kiapour and Nematollahi, 2011) Let $\Gamma$ be a class of priors, $P$ be a decision rule and $\rho(\pi, P)$ be the posterior risk given by (3.1).

(i) The conditional $\Gamma$-minimax (CGM) estimator $P_{\Gamma}^{C G M}$ is defined as

$$
\sup _{\pi \in \Gamma} \rho\left(\pi, P_{\Gamma}^{C G M}\right)=\inf _{P \in D} \sup _{\pi \in \Gamma} \rho(\pi, P) .
$$

As the name suggests, statisticians are interested in devising an estimator, conditional on observations, that minimizes posterior expected loss $\rho(\pi, P)$ over $\Gamma$.

(ii) The most stable (MS) estimator $P_{\Gamma}^{M S}$ is defined as

$$
\sup _{\pi \in \Gamma} \rho\left(\pi, P_{\Gamma}^{M S}\right)-\inf _{\pi \in \Gamma} \rho\left(\pi, P_{\Gamma}^{M S}\right)=\inf _{P \in D}\left[\left(\sup _{\pi \in \Gamma} \rho(\pi, P)-\inf _{\pi \in \Gamma} \rho(\pi, P)\right)\right] .
$$

The MS estimator minimizes the oscillation of $\rho(\pi, P)$ over $\Gamma$ w.r.t. $P \in D$.

(iii) The posterior regret $\Gamma$-minimax (PRGM) estimator $P_{\Gamma}^{P R G M}$ is defined as

$$
\sup _{\pi \in \Gamma} R\left(P_{\Gamma}^{P R G M}, P^{\pi}\right)=\inf _{P \in D} \sup _{\pi \in \Gamma} R\left(P, P^{B}\right),
$$

where $R\left(P, P^{\pi}\right)=\rho(\pi, P)-\rho\left(\pi, P^{B}\right)$ is the posterior regret, which measures the loss of optimality due to choosing the estimator $P\left(\mathbf{x}^{n}\right)$ instead of the optimal Bayes estimator $P^{B}\left(\mathbf{x}^{n}\right)$. 
(iv) The least sensitive (LS) estimator $P_{\Gamma}^{L S}$ is defined as

$$
\sup _{\pi \in \Gamma} \frac{R\left(P_{\Gamma}^{L S}, P^{B}\right)}{\rho\left(\pi, P^{\pi}\right)}=\inf _{P \in D} \sup _{\pi \in \Gamma} \frac{R\left(P, P^{B}\right)}{\rho\left(\pi, P^{\pi}\right)} .
$$

Note that, this measure is scale invariant and minimizes the relative error in the posterior expected loss $\rho(\pi, P)$ when considering an estimator $P\left(\mathbf{x}^{n}\right)$ instead of the optimal Bayes estimator $P^{\pi}\left(\mathbf{x}^{n}\right)$.

To obtain robust Bayes estimators of $P(\theta)$, consider a class of prior distributions for $\theta$ as

$$
\Gamma=\left\{\pi_{\alpha_{0}, \beta}, \beta \in\left[\beta_{1}, \beta_{2}\right] \subset R^{+}, \alpha=\alpha_{0}>0\right\}
$$

In the following theorem, we obtain the CGM, MS, PRGM and LS premium estimators of $P(\theta)$ under the loss function (2.2) in the class $\Gamma$ of prior distributions.

Theorem 3.2. Let $X_{1}, X_{2}, \ldots$ be a sequence of random variables which are conditionally independent given $\theta$, and suppose that $X_{i}$ given $\theta$ has $\operatorname{EXP}(\theta)$-distribution. Suppose that $X^{n}=x^{n}$. Then, the CGM, MS, PRGM and LS premium estimators of $P(\theta)=\frac{u}{\theta}$ under the loss function (2.2) in the class $\Gamma$ are equal to

$$
P_{\Gamma}^{R B}\left(x^{n}\right)=u e^{-\psi(n+\alpha)} \sqrt{\left(T+\beta_{1}\right)\left(T+\beta_{2}\right)} .
$$

Proof. To find CGM premium estimator, we obtain the posterior risk of $P\left(\mathbf{x}^{n}\right)$ as

$$
\begin{aligned}
\rho(\pi, P) & =\ln ^{2} P+E\left[\ln ^{2} P(\theta) \mid \mathbf{x}^{n}\right]-2 \ln P E\left[\ln P(\theta) \mid \mathbf{x}^{n}\right] \\
& =A^{*}+\ln ^{2} b-2 \ln b[\psi(n+\alpha)-\ln u+\ln P]+\ln ^{2} P+2 \ln P[\psi(n+\alpha)-\ln u] \\
& =\rho\left(\pi_{b}, P\right),
\end{aligned}
$$

where $A^{*}=\psi^{\prime}(n+\alpha)+[\ln b-\psi(n+\alpha)]^{2}$ and $b=T+\beta$. Note that

$$
\frac{\partial}{\partial b} \rho\left(\pi_{b}, P\right)=2 \frac{[\ln b-\psi(n+\alpha)+\ln u+\ln P]}{b},
$$

and

$$
\frac{\partial^{2}}{\partial b^{2}} \rho\left(\pi_{b}, P\right)=2 \frac{[1-\ln b+\psi(n+\alpha)-\ln u-\ln P]}{b^{2}} .
$$


Thus, $\rho\left(\pi_{b}, P\right)$ is not a strictly convex function of $b$, but has a minimum at $b_{\text {min }}=$ $P e^{\psi(n+\alpha)-u}$. The function $l(P)=\rho\left(\pi_{b_{1}}, P\right)-\rho\left(\pi_{b_{2}}, P\right)$ is an increasing and continuous function of $P$. Also, we have

$$
l(P)=0 \text { iff } P=u e^{-\psi(n+\alpha)} \sqrt{b_{1} b_{2}}=P^{*},
$$

where $b_{i}=T+\beta_{i}, \quad i=1,2$. Hence,

$$
\sup _{b \in\left[b_{1}, b_{2}\right]} \rho\left(\pi_{b}, P\right)= \begin{cases}\rho\left(\pi_{b_{1}}, P^{*}\right) & P \geqslant P^{*} \\ \rho\left(\pi_{b_{2}}, P^{*}\right) & P \leqslant P^{*}\end{cases}
$$

Note that $\rho\left(\pi_{b}, P\right)$ is a strictly convex function of $P$ and has a minimum at $P^{B}=$ $u b e^{-\psi(n+\alpha)}$. Since $P_{\pi_{b_{1}}}^{B}=u b_{1} e^{-\psi(n+\alpha)}<P^{*}$, then, for all $P \geqslant P^{*}, \inf _{P \geqslant P^{*}} \rho\left(\pi_{b_{1}}, P\right)=\rho\left(\pi_{b_{1}}, P^{*}\right)$. Moreover, $P_{\pi_{b_{2}}}^{B}=u b_{2} e^{-\psi(n+\alpha)}>P^{*}$, then, for all $P \leqslant P^{*}, \inf _{P \leqslant P^{*}} \rho\left(\pi_{b_{2}}, P\right)=\rho\left(\pi_{b_{2}}, P^{*}\right)$. This implies that

$$
\inf _{b \in\left[b_{1}, b_{2}\right]} \sup _{\pi \in \Gamma} \rho\left(\pi_{b}, P\right)=\rho\left(\pi_{b_{1}}, P^{*}\right)=\rho\left(\pi_{b_{2}}, P^{*}\right)
$$

Consequently, we have

$$
\begin{aligned}
P_{\Gamma}^{C G M}=P^{*} & =u e^{-\psi(n+\alpha)} \sqrt{b_{1} b_{2}}, \\
& =u e^{-\psi(n+\alpha)} \sqrt{\left(T+\beta_{1}\right)\left(T+\beta_{2}\right)} .
\end{aligned}
$$

To find the MS premium estimator, we obtain the $\inf _{\pi \in \Gamma} \rho(\pi, P)$. Since $\inf _{\pi_{\beta \in \Gamma}} \rho\left(\pi_{\beta}, P\right)=$ $\psi^{\prime}(n+\alpha)$, which does not depend on the value of $P$,

$$
\inf _{P \in D}\left[\sup _{\pi \in \Gamma} \rho(\pi, P)-\inf _{\pi \in \Gamma} \rho(\pi, P)\right]=\inf _{P \in D} \sup _{\pi \in \Gamma} \rho(\pi, P)-\psi^{\prime}(n+\alpha),
$$

which implies that $P_{\Gamma}^{M S}=P_{\Gamma}^{C G M}$.

To find the $P R G M$ and $L S$ premium estimators, we obtain the posterior risk of Bayes premium estimator $P^{B}\left(\mathbf{x}^{n}\right)=u(T+\beta) e^{-\psi(n+\alpha)}$ under the SLEL function which is given by

$$
\rho\left(\pi, P^{B}\right)=\psi^{\prime}(n+\alpha) .
$$

Since the posterior risk of the Bayes estimator does not depend on the value of $\beta$, from Definitions 1-3, the estimators $P_{\Gamma}^{P R G M}$ and $P_{\Gamma}^{L S}$ are the same as $\hat{P}_{\Gamma}^{C G M}$. 


\section{Bayesian Predictors of the Claim Size}

In many practical situations, an insurance company needs to predict the future claims in order to evaluate premiums, to assess its financial situation, probabilities of ruin, automobile insurance, loss reserving, and etc. In this section, we study the Bayesian prediction of the future claim size $X_{n+1}$ from the past observation $\mathbf{x}^{n}=\left(x_{1}, \ldots, x_{n}\right)$ of claims $\mathbf{X}^{n}=\left(X_{1}, \ldots, X_{n}\right)$. Given $\mathbf{X}^{n}=\mathbf{x}^{n}$, the predictive distribution is given by

$$
h\left(x_{n+1} \mid \mathbf{x}^{n}\right)=\int_{\Theta} f\left(x_{n+1} \mid \theta\right) \pi\left(\theta \mid \mathbf{x}^{n}\right) d \theta .
$$

We are interested in prediction of $X_{n+1}$ by $P_{n+1}=\hat{X}_{n+1}$ from the past observations $\mathbf{x}^{n}$ under the following prediction loss function

$$
L\left(X_{n+1}, P_{n+1}\right)=\left(\ln P_{n+1}-\ln X_{n+1}\right)^{2}=\ln ^{2}\left[\frac{P_{n+1}}{X_{n+1}}\right], \quad P_{n+1} \in D .
$$

\subsection{Bayes, E-Bayes and Robust Bayes Predictor of the Claim Size}

The Bayes predictor of the claim size is obtained by minimizing the posterior risk

$$
\begin{aligned}
\rho\left(\pi, P_{n+1}\right) & =E\left[L\left(X_{n+1}, P_{n+1}\right) \mid \mathbf{X}^{n}=\mathbf{x}^{n}\right] \\
& =\ln ^{2} P_{n+1}\left(\mathbf{x}^{n}\right)+E\left[\ln ^{2} X_{n+1} \mid \mathbf{x}^{n}\right]-2 \ln P_{n+1}\left(\mathbf{x}^{n}\right) E\left[\ln X_{n+1} \mid \mathbf{x}^{n}\right],
\end{aligned}
$$

over $P_{n+1} \in D$, which gives us

$$
\hat{P}_{n+1}^{B}\left(\mathbf{x}^{n}\right)=e^{E\left[\ln X_{n+1} \mid \mathbf{X}^{n}=\mathbf{x}^{n}\right]} .
$$

Combining the likelihood based on the model in (2.1) and the prior (3.3), the posterior distribution of $\theta$ given $\mathbf{X}^{n}=\mathbf{x}^{n}$ is $\operatorname{Gamma}\left(n+\alpha,(T+\beta)^{-1}\right)$. Therefore, $E\left[\ln X_{n+1} \mid \mathbf{x}^{n}\right]=E\left\{E\left[\ln X_{n+1} \mid \theta\right] \mid \mathbf{x}^{n}\right\}=E\left[\psi(1)-\ln \theta \mid \mathbf{x}^{n}\right]=\ln u_{1}-\psi(n+\alpha)+\ln (T+\beta)$, where $u_{1}=e^{\psi}(1)$. So, the Bayes predictor of the claim size $X_{n+1}$ under the loss function (4.1) is given by

$$
\hat{P}_{n+1}^{B}\left(\mathbf{x}^{n}\right)=u_{1}(T+\beta) e^{-\psi(n+\alpha)} .
$$

The E-Bayes and robust Bayes predictors of the claim size can then be defined by replacing $\rho(\pi, P)$ in (3.1) with $\rho\left(\pi, P_{n+1}\right)$ in (4.2) and $P^{B}$ in (3.2) by $\hat{P}_{n+1}^{B}$ in (4.3), respectively.

Note that the Bayes predictor of the claim size $\hat{P}_{n+1}^{B}$ in (4.3) is similar to the Bayes premium estimator $P^{B}\left(\mathbf{x}^{n}\right)$ given by (3.4) when $u=u_{1}$. Then, E-Bayes predictors of the 
claim size $\hat{P}_{n+1}^{E B_{i}}, \quad i=1,2,3$, under three prior distributions (3.7) are the same as E-Bayes premium estimators (3.8). Moreover, the robust Bayes predictors of the claim size $\hat{P}_{n+1}^{R B}$ and the robust Bayes premium estimators (3.11) under the loss function (4.1) in the class $\Gamma$ of prior distributions are equivalent.

\section{Numerical Comparisons of Predictors}

We provide a numerical study to illustrate the results obtained in Section 4 . To do this, a prequential analysis (Dawid and Vovk, 1999; Kiapour and Nematollahi, 2011) is employed by using a simulation to compare the Bayes, E-Bayes and robust Bayes predictors. Let $\hat{P}_{n+1}^{k}, k=1,2,3,4,5$, stand for $\hat{P}_{n+1}^{B}\left(\mathbf{x}^{n}\right)$ with $\alpha=1$ and $\beta=5 ; \hat{P}_{n+1}^{E B_{i}}\left(\mathbf{x}^{n}\right), i=$ $2,3,4$, with $c=1,5,9$ and $P_{n+1}^{R B}\left(\mathbf{x}^{n}\right)$ with $\alpha=1, \beta \in[1,5]$.

The simulation study proceeds as follows.

1. Generate the sample $x_{1}, \ldots, x_{n}$ from the $\operatorname{EXP}(\theta)$-distribution with $\theta=3$.

2. Generate $x_{n+1}$ from the distribution in step 1 and calculate a predictor $\hat{P}_{n+1}^{k}\left(\mathbf{x}^{n}\right)$ based on $\mathbf{x}^{n}=\left(x_{1}, \ldots, x_{n}\right)$ for $x_{n+1}$.

3. Calculate the prediction error for the observation $x_{n+1}$, i.e. $\left(\ln \hat{P}_{n+1}^{k}\left(\mathbf{x}^{n}\right)-\ln x_{n+1}\right)^{2}$.

4. Increase $n$ by 1 and repeat Steps 2 and 3 until $n=m$, where $m=5,10,30,50,100$.

5. Average all of the one-step-ahead prediction errors calculated in Step 3. This yields the average prediction error (APE) given by

$$
A P E=\frac{1}{m} \sum_{n=1}^{m}\left(\ln \hat{P}_{n+1}^{k}\left(\mathbf{x}^{n}\right)-\ln x_{n+1}\right)^{2},
$$

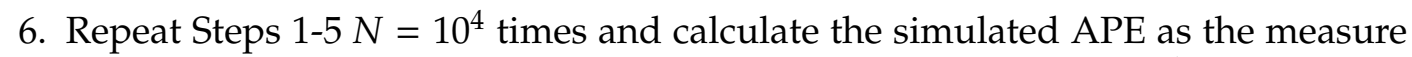
of prediction error by averaging of the APEs given in (5.1) over $10^{4}$ repetitions.

The values of simulated APE of the predictors are summarized in Table 1 . The predictors with good performance are bold. The following points can be obtained from Table 1:

- For $c=1$, the E-Bayes predictors work better than the Bayes and robust Bayes predictors. For $c=5$, the E-Bayes predictor performs better than all of other predictors. 
Table 1: Simulated APE for the Bayes premium predictor, the E-Bayes premium predictors and the robust Bayes premium predictor of $X_{n+1}$ for selected values of $c=1,5,9$ and $m=5,10,30,50,100$ over $\Gamma$.

\begin{tabular}{cccccc} 
& \multicolumn{4}{c}{$c=1$} & \\
\cline { 2 - 5 }$m$ & $\hat{P}_{n+1}^{B}$ & $\hat{P}_{n+1}^{E B_{1}}$ & $\hat{P}_{n+1}^{E B_{2}}$ & $\hat{P}_{n+1}^{E B_{3}}$ & $\hat{P}_{n+1}^{R B}$ \\
\hline 5 & 4.69564 & $\mathbf{1 . 7 9 1 8 0}$ & $\mathbf{1 . 8 0 0 0 9}$ & $\mathbf{1 . 8 5 6 4 8}$ & 3.01745 \\
10 & 3.73655 & $\mathbf{1 . 7 9 5 7 1}$ & $\mathbf{1 . 7 9 8 8 7}$ & $\mathbf{1 . 8 3 0 0 4}$ & 2.58523 \\
30 & 2.54130 & $\mathbf{1 . 7 1 5 2 9}$ & $\mathbf{1 . 7 1 4 7 1}$ & $\mathbf{1 . 7 2 4 5 8}$ & 2.03260 \\
50 & 2.23217 & $\mathbf{1 . 7 0 1 5 2}$ & $\mathbf{1 . 7 0 0 9 8}$ & $\mathbf{1 . 7 0 6 9 0}$ & 1.90300 \\
100 & 1.95739 & $\mathbf{1 . 6 7 9 3 6}$ & $\mathbf{1 . 6 7 9 0 8}$ & $\mathbf{1 . 6 8 2 0 4}$ & 1.78360 \\
\hline
\end{tabular}

\begin{tabular}{cccccc}
\multicolumn{5}{c}{$c=5$} \\
\cline { 2 - 5 }$m$ & $\hat{P}_{n+1}^{B}$ & $\hat{P}_{n+1}^{E B_{1}}$ & $\hat{P}_{n+1}^{E B_{2}}$ & $\hat{P}_{n+1}^{E B_{3}}$ & $\hat{P}_{n+1}^{R B}$ \\
\hline 5 & 4.75498 & $\mathbf{2 . 5 3 1 4 0}$ & 3.13101 & 3.70633 & 3.06508 \\
10 & 3.72409 & $\mathbf{2 . 2 0 8 3 5}$ & 2.59508 & 2.98351 & 2.57612 \\
30 & 2.54926 & $\mathbf{1 . 8 7 4 5 1}$ & 2.03383 & 2.20355 & 2.03971 \\
50 & 2.23047 & $\mathbf{1 . 7 9 5 0 4}$ & 1.89548 & 2.00424 & 1.90187 \\
100 & 1.963553 & $\mathbf{1 . 7 3 3 0 5}$ & 1.78507 & 1.84224 & 1.78966 \\
\hline
\end{tabular}

\begin{tabular}{cccccc} 
& \multicolumn{5}{c}{$c=9$} \\
\cline { 2 - 5 }$m$ & $\hat{P}_{n+1}^{B}$ & $\hat{P}_{n+1}^{E B_{1}}$ & $\hat{P}_{n+1}^{E B_{2}}$ & $\hat{P}_{n+1}^{E B_{3}}$ & $\hat{P}_{n+1}^{R B}$ \\
\hline 5 & 4.73507 & 3.46847 & 4.43625 & 5.30060 & $\mathbf{3 . 0 5 5 4 6}$ \\
10 & 3.68968 & 2.80066 & 3.47495 & 4.10314 & $\mathbf{2 . 5 5 0 1 6}$ \\
30 & 2.56212 & 2.14577 & 2.45808 & 2.76811 & $\mathbf{2 . 0 4 9 8 3}$ \\
50 & 2.22813 & 1.95835 & 2.15999 & 2.36506 & $\mathbf{1 . 9 0 0 2 1}$ \\
100 & 1.96314 & 1.81826 & 1.9261 & 2.03804 & $\mathbf{1 . 7 8 8 8 7}$ \\
\hline
\end{tabular}

Moreover, for $c=9$, the robust Bayes predictor is better than Bayes and E-Bayes predictors.

- The E-Bayes and robust Bayes predictors work better than the Bayes predictor.

- The simulated APE are more close to each other for large values of $c$.

- The simulated APE decreases as the sample size $m$ increases.

\section{A Real Data Analysis}

In this section, we use a real data-set to illustrate the application of the prediction results. The aircraft insurance data presented in Table 2 from the central insurance of Iran are the paid claims of aircraft insurance (per ten Billion Rial) for 10 years. To 
Table 2: The claims size of aircraft insurance

\begin{tabular}{ccccccccccc}
\hline year & 2006 & 2007 & 2008 & 2009 & 2010 & 2011 & 2012 & 2013 & 2014 & 2015 \\
\hline claim & 18.93 & 10.11 & 22.31 & 32.97 & 21.98 & 11.96 & 14.86 & 6.94 & 57.2 & 68.07 \\
\hline
\end{tabular}

Table 3: The predicted values for claim $X_{10}$.

\begin{tabular}{ccccc}
\hline$\hat{P}_{n+1}^{B}$ & $\hat{P}_{n+1}^{E B_{1}}$ & $\hat{P}_{n+1}^{E B_{2}}$ & $\hat{P}_{n+1}^{E B_{3}}$ & $\hat{P}_{n+1}^{R B}$ \\
\hline 51.8971 & 52.6757 & 53.0702 & 53.4648 & 53.6264 \\
\hline
\end{tabular}

study the goodness of the fit of the exponential model, a Kolmogorov-Smirnov test with the test statistic of 0.2302 and corresponding p-value of 0.5878 suggests that the exponential model with mean $\frac{1}{\theta}=26.533$ can be used to analyze this data-set.

Our interest is to predict the claim in year 2015, i.e., $X_{10}=68.07$. We consider Gamma(1,0.04)-prior with mean 25 which is close to the mean of exponential distribution 26.533. For this purpose, we obtained the Bayes predictor $\hat{P}_{n+1}^{B}\left(\mathbf{x}^{n}\right)$, E-Bayes predictors $\hat{P}_{n+1}^{E B_{i}}\left(\mathbf{x}^{n}\right), i=2,3,4$, with $c=9$ and the robust Bayes predictor $P_{n+1}^{R B}\left(\mathbf{x}^{n}\right)$ with $\alpha=0.9$ and $\beta \in[0.04,9]$ and summarized them in Table 3 . We observe from this table that all predictors are close to each other and the value of the robust Bayes predictor is closer to the true value 68.07 than Bayes and E-Bayes predictors. Moreover, we see that E-Bayes predictors are very close to each other.

\section{Concluding Remarks}

The Bayes estimator is obtained by choosing an explicit prior distribution over the parameter of interest. In practical situations, more frequently, the prior knowledge is vague and any elicited prior distribution is only an approximation to the true one. So, the E-Bayesian and the robust Bayesian analysis can be employed to handle the uncertainty in specifying the prior distribution by considering a class of priors instead of a single prior. Our aim of this paper was to compare Bayes, E-Bayes and robust Bayes estimators and predictors under the SLEL function. We first considered a prior for $\theta$ with hyperparameters $\alpha$ and $\beta$ and obtained the Bayes estimator. Considering the robustness of Bayesian estimator, the thinner tailed prior distribution often reduces the robustness of Bayesian estimator. Therefore, we investigated the performance of E-Bayes and robust Bayes estimators or predictors for selected values of $c$ (an upper bound for $\beta$ ) in comparison with the Bayes estimator. Our findings in a simulation 
study for comparing the predictors showed that robust Bayes and E-Bayes predictors work better than the Bayes predictor. We also considered the aircraft insurance claim data of Iran between years 2006-2015 and applied our results for predicting the claim for year 2015. In this case, the robust Bayes predictor performed better than other predictors for predicting the true value of the claim.

\section{Acknowledgements}

The author thanks the Editor, the Associate Editor and the two referees for careful reading and for the comments which greatly improved the paper.

\section{References}

Arias-Nicolas, J. P., Martin, J., Ruggeri, F., Suarez-Llorens, A. (2009). Optimal actions in problems with convex loss function. International Journal of Approximate Reasoning, 50, 303-314.

Berger, J. O. (1984). The robust Bayesian viewpoint (with discussion). In Robustness of Bayesian Analysis (J. Kadane ed.), North Holland, Amsterdam.

Berger, J. O. (1985). Statistical Decision Theory and Bayesian Analysis, Second ed., springerVerlag, New York.

Betro, B., Ruggeri, F. (1992). Conditional $\Gamma$-minimax actions under convex losses, Communications in Statistics-Theory and Methods, 21, 1051-1066.

Boratynska, A. (2008). Posterior regret gamma minimax estimation of insurance premium in collective risk model. Astin Bulletin, 38(1), 277-291.

Brown, L. D. (1968). Inadmisibility of the usual estimator of scale parameters in problems with unknown location and scale parameters. Annals of Mathematical Statistics, $39,29-48$.

Dawid, A. P. and Vovk, V. G. (1999). Prequential probability: principals and properties. Bernoulli, 5(1), 125-162.

Gomez-Deniez, E., Perez, J. M., Vazquez-Polo, F.J. (2006). On the use of posterior regret $\Gamma$-minimax actions to obtain credibility premiums. Insurance: Mathematics and Economics, 39, 115-121. 
Bayes, E-Bayes and Robust Bayes Premium Estimation and Prediction...

Goovaerts, M. J., Kaas, R., van Heerwaarden, A. E., Bauwelinckx, T. (1990). Effective actuarial methods, North Holland, Amsterdam.

Jaheen, Z. F. and Okasha, H. M. (2011). E-Bayesian estimation for the burr type XII model based on type II censoring, Applied Mathematical Modeling, 35, 4730-4737.

Han, M. (1997). The structure of hierarchical prior distribution and its applications, Chinese Operations Research and Management Science, 6, 31-40.

Han, M. (2007). E-Bayesian estimation of failure probability and its application, Mathematical and Computer Modelling, 45, 1272-1279.

Han, M. (2009). E-Bayesian estimation and hirarchical Baysian estimation of failure rate, Applied Mathematical Modeling, 33, 1915-1922.

Han, M. (2011). E-Bayesian estimation and hirarchical Baysian estimation of failure probability, Communications in Statistics-Theory and Methods, 40, 3303-3314.

Heilmann, W. (1989). Decision theoretic foundations of credibility theory. Insurance: Mathematics and Economics, 8(1), 77-95.

Kiapour, A., and Nematollahi, N. (2011). Robust Bayesian prediction and estimation under a square error loss function, Statistics and Probability Letters, 81, 1717-1724.

Klugman, S. A., 1992. Bayesian Statistics in actuarial science: with emphasis on credibility. Kluwer, Boston.

Meczarski, M. (1993). Stability and conditional gamma-minimaxity in Bayesian inference, Mathematicae, 22, 117-122.

Naghizadeh Qomi, M., and Barmodeh, L. (2015). Shrinkage testimation in exponential distribution based on records under asymmetric squared log error, Journal of Statistical Research of Iran, 12, 225-238.

Rios Insua, D., Ruggeri, F., Vidakovic, B. (1995). Some results on posterior regret $\Gamma-$ minimax estimation, Statistics and Decisions, 13, 315-331.

Rios, S., Martin, J., Rios, D., Ruggeri, F. (1999). Bayesian forecasting for accident proneness evaluation, Scandinavian Actuarial Journal, 2, 134-156.

Sanjari Farsipour, N., Zakerzadeh, H. (2005). Estimation of a Gamma scale parameter under asymmetric squard log error loss. Communications in Statistics-Theory and Methods, 34, 1127-1135. 